\title{
Studies of exotic hadrons by high-energy exclusive reactions
}

\author{
H. Kawamura ${ }^{a}$, S. Kumano ${ }^{a, b}$, and T. Sekihara ${ }^{a}$ \\ ${ }^{a}$ KEK Theory Center, Institute of Particle and Nuclear Studies, KEK \\ 1-1, Ooho, Tsukuba, Ibaraki, 305-0801, Japan \\ ${ }^{b}$ J-PARC Branch, KEK Theory Center, Institute of Particle and Nuclear Studies, KEK \\ and Theory Group, Particle and Nuclear Physics Division, J-PARC Center \\ 203-1, Shirakata, Tokai, Ibaraki, 319-1106, Japan
}

(Received July 15, 2013)

\begin{abstract}
We investigate the possibility of clarifying internal structure of exotic hadrons by high-energy exclusive reactions. In particular, the constituent-counting rule could be used for determining the internal configuration in large-angle exclusive scattering. As an example, we show the cross section $\pi^{-}+p \rightarrow K^{0}+\Lambda(1405)$ in comparison with the one for the ground-state $\Lambda$ production $\pi^{-}+p \rightarrow K^{0}+\Lambda$. The counting rule indicates that the cross section scales as $s^{8} d \sigma / d t=$ constant if $\Lambda(1405)$ is an ordinary three-quark baryon, whereas it is $s^{10} d \sigma / d t=$ constant if $\Lambda(1405)$ is a five-quark baryon. Here, $s$ and $t$ are Mandelstam variables. Such experiments could be possible at J-PARC, LEP, JLab, CERNCOMPASS, and other high-energy facilities.
\end{abstract}

KEYWORDS: exotic hadron, exclusive reaction, perturbative QCD

\section{Introduction}

Hadrons are classified into two categories, mesons and baryons. According to a naive quark model, the mesons and baryons have the configurations of $q \bar{q}$ and $q q q$, respectively. Since the underlying fundamental theory of strong interactions (QCD) does not prohibit different forms of configurations such as tetraquark $(q q \bar{q} \bar{q})$ and pentaquark $(q q q q \bar{q})$, exotic hadrons have been searched experimentally for a long time since 1960's. It is fortunate that some exotic hadron candidates have been reported in the last several years particularly by the Belle and BaBar collaborations. Nevertheless, it is rather difficult to find an undoubted evidence because similar theoretical results could be obtained for global quantities such as spins, parities, masses, and decay widths even by conventional $q \bar{q}$ and $q q q$ models although there are some indications, for example, that $f_{0}(980)$ and $a_{0}(980)$ could be tetraquark or $K \bar{K}$ molecule and that $\Lambda$ (1405) could be a pentaquark or $\bar{K} N$ molecule [1].

We have been investigating new approaches to the exotic-hadron studies by using high-energy hadron reaction processes [2,3], where quark and gluon degrees of freedom appear. First, we consider the two-photon process $\gamma^{*} \gamma \rightarrow h \bar{h}$ for probing internal structure of the hadron $h$ such as $f_{0}(980)$ and $a_{0}(980)$ [2]. Exotic signatures appear in generalized distribution amplitudes (GDAs) which can be measured in the two-photon process. The GDAs correspond to the GPDs (generalized parton distributions) by the $s$ - $t$ channel crossing. The studies of the GDAs together with the GPDs should shed light on a new aspect of exotic-hadron physics for future developments in clarifying the existence of exotics and their internal quark-gluon configurations [2].

Next, we propose to use hard exclusive production of an exotic hadron, by taking $\Lambda(1405)$ as an example, for finding its internal quark configuration [3]. In particular, the cross section for the exclusive process $\pi^{-}+p \rightarrow K^{0}+\Lambda(1405)$ is estimated at the scattering angle $\theta_{c m}=90^{\circ}$ in the center-of-mass frame by using exiting experimental data. We suggest that the internal quark configuration of $\Lambda(1405)$ should be determined by the asymptotic scaling behavior of the cross section in comparison with the ground-state $\Lambda$ production. Such measurements will be possible, for example, 
by using the high-momentum beamline of J-PARC and at LEPS, JLab, and CERN-COMPASS. Since the first project of two-photon process has not been completed yet, we discuss the second one on the constituent counting rule [3] in the following.

\section{Constituent-counting rule for hard exclusive processes}

We consider a large-angle exclusive scattering $a+b \rightarrow c+d$. Its cross section is given by

$$
\frac{d \sigma_{a b \rightarrow c d}}{d t} \simeq \frac{1}{16 \pi s^{2}} \sum_{p o l}\left|M_{a b \rightarrow c d}\right|^{2},
$$

where $s$ and $t$ are Mandelstam variables defined by the momenta $p_{i}(i=a, b, c, d)$ as $s=\left(p_{a}+p_{b}\right)^{2}$ and $t=\left(p_{a}-p_{c}\right)^{2}$, and the matrix element is expressed as [4]

$$
\begin{aligned}
M_{a b \rightarrow c d}=\int\left[d x_{a}\right]\left[d x_{b}\right]\left[d x_{c}\right]\left[d x_{d}\right] \phi_{c}\left(\left[x_{c}\right]\right) \phi_{d}\left(\left[x_{d}\right]\right) \\
\times H_{a b \rightarrow c d}\left(\left[x_{a}\right],\left[x_{b}\right],\left[x_{c}\right],\left[x_{d}\right], Q^{2}\right) \phi_{a}\left(\left[x_{a}\right]\right) \phi_{b}\left(\left[x_{b}\right]\right) .
\end{aligned}
$$

Here, $H_{a b \rightarrow c d}$ is the partonic scattering amplitude, $\phi_{i}$ is the light-cone distribution amplitude of the hadron $i(i=a, b, c, d)$ as illustrated in Fig. 1 , and $[x]$ indicates a set of the light-cone momentum fractions of partons in a hadron: $x_{i}=p_{i}^{+} / p^{+}$where $p_{i}$ and $p$ are $i$-th parton and hadron momenta, respectively.

For a particle $h$ with $N_{h}$ constituents, the state vector could be written as $|h\rangle=\sqrt{N_{h}}\left|n_{h}\right\rangle$. The state vector of a hadron is normalized as $\left\langle h\left(p^{\prime}\right) \mid h(p)\right\rangle=2 p^{0}(2 \pi)^{3} \delta^{(3)}\left(\vec{p}-\vec{p}^{\prime}\right)$, so that its mass dimension is $[1 / M]$. If the state vector of each constituent has the mass dimension $[1 / M]$, the normalization factor has the mass dimension $\left[\sqrt{N_{h}}\right]=\left[M^{n_{h}-1}\right]$. The normalization factors are expressed by soft constants of the order of a hadron mass, so that other mass-dimension factors come from hard momenta in the reaction. The variable $s$ could be chosen as the only hard scale in the large-angle exclusive reaction, then the cross section becomes [5]

$$
\frac{d \sigma_{a b \rightarrow c d}}{d t}=\frac{1}{s^{n-2}} f_{a b \rightarrow c d}(t / s)
$$

where $f(t / s)$ is a scattering-angle dependent function, and the factor $n$ is defined by $n=n_{a}+n_{b}+n_{c}+$ $n_{d}$. Because the cross section scales as $1 / s^{n-2}$ with the number of constituents, this scaling behavior is called the constituent-counting rule.

This counting rule was investigated in perturbative QCD [4]. Due to the nature of the large-angleexclusive reaction, quarks should share large momenta so that they should stick together to form a hadron by exchanging hard gluons as shown in Fig. 2. Assigning hard momentum factors for the internal quark and gluon propagators and considering the hard factors for external quarks, we could explain the constituent-counting rule in perturbative QCD although there are some complications from disconnected diagrams [3].

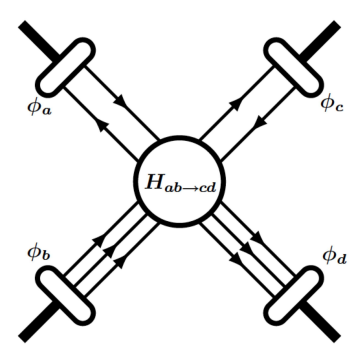

Fig. 1. Exclusive processes $a+b \rightarrow c+d$.

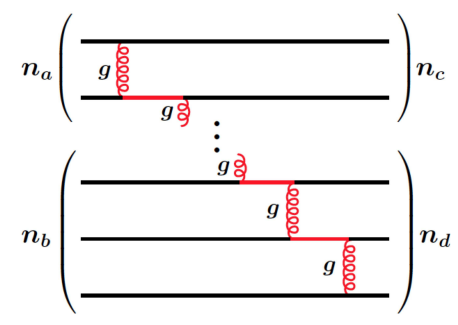

Fig. 2. Hard gluon exchange process. 


\section{Exclusive production of exotic hadron for probing its internal structure}

The constituent-counting rule has been investigated experimentally at BNL and JLab, and their data support the scaling predicted by the counting rule [6]. As an example, the cross section $s^{7} d \sigma / d t$ is shown in Fig. 3 at $\theta_{c . m .}=90^{\circ}$ for the process $\gamma+p \rightarrow \pi^{+}+n$, where the total number of constituents is nine, as a function of the center-of-mass energy $\sqrt{s}$. At low energies, resonancelike bumps appear, whereas the cross section multiplied by $s^{7}$ is constant at high energies, which agrees with the scaling of the counting rule. It is also interesting to find that the transition from hadron degrees of freedom to quark ones seems to be apparent at $\sqrt{s}=2.5 \mathrm{GeV}$.

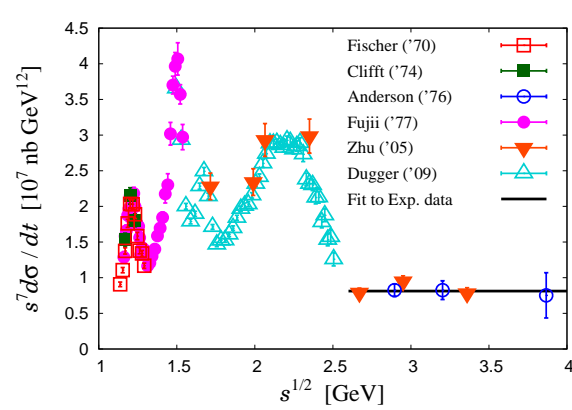

Fig. 3. Cross section of $\gamma+p \rightarrow \pi^{+}+n$.

We use the idea of constituent-counting rule for probing internal structure of exotic hadron candidates, especially $\Lambda(1405)$. The $\Lambda(1405)$ is a baryon resonance with isospin 0 , spin-parity $(1 / 2)^{-}$, strangeness -1 , mass $1405.1 \mathrm{MeV}$, and width $50 \mathrm{MeV}$. The naive quark model treats the $\Lambda$ (1405) as an $u d s$ three-quark system, but the quark model cannot explain that the $\Lambda(1405)$ mass is much ligher than that of the lightest nucleon resonance with $(1 / 2)^{-}, N(1535)$. Therefore, instead of an $u d s$ quark system, it is thought as an exotic hadron such as a $\bar{K} N$ molecule [1].

Before discussing the case of $\Lambda(1405)$, we first consider the case of ground-state $\Lambda$, which is expected to be an ordinary three-quark system. There are measurements on the cross section of $\pi^{-}+p \rightarrow K^{0}+\Lambda$ [7]. From the data, we extracted the cross sections at $\theta_{c . m} .=90^{\circ}$, and they are shown in Fig. 4. The data are compared with theoretical estimates calculated for the processes $\pi^{-}+p \rightarrow N\left(N^{*}\right) \rightarrow K^{0}+\Lambda$ by including thirteen $s$-channel $N^{*}$ resonances: $N(1535), N(1650)$, $N(1440), N(1710), N(1750), N(1720), N(1520), N(1675), N(1680), N(1990), N(2190), N(2250)$, and $N(2220)$ [7]. There are two curves depending on the parameter choice. They agree with the data, which indicates that the $\Lambda$-production cross sections at low energies should be described by the formation processes of the $s$-channel $N^{*}$ resonances. In order to investigate the high-energy region, the cross section multiplied by $s^{8}$ is shown in Fig. 5 by considering the number of total constituents $(n=10)$. Although the experimental data are not very accurate, there is a tendency that $s^{8} d \sigma / d t=$ constant at $\sqrt{s}>2 \mathrm{GeV}$. In fact, fitting the data by the function $d \sigma / d t=$ (constant) $\times s^{2-n}$, we obtain the scaling factor [3]

$$
n=10.1 \pm 0.6 \text {. }
$$

It is consistent with the counting rule, which encourages us to investigate the scaling behavior of exotic hadron production.

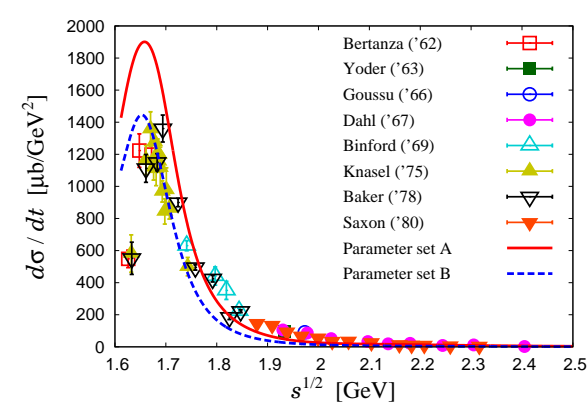

Fig. 4. Cross section of $\pi^{-}+p \rightarrow K^{0}+\Lambda$ [3].

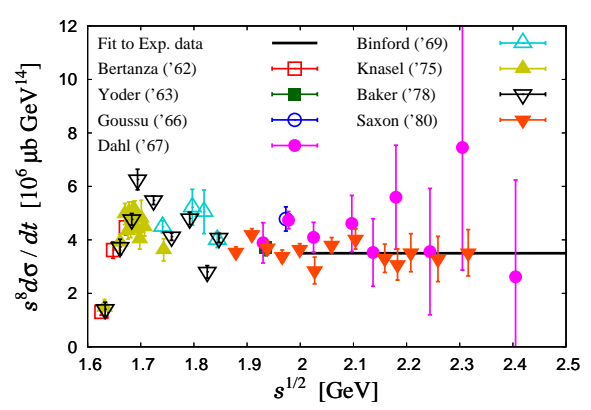

Fig. 5. Cross section of $\pi^{-}+p \rightarrow K^{0}+\Lambda$ [3]. 
Next, we study the cross section of $\pi^{-}+p \rightarrow K^{0}+\Lambda$ (1405) for finding the internal structure of $\Lambda$ (1405). However, both experimental and theoretical studies are very limited. In fact, there is only one experimental measurement [8] as shown in Fig. 6. At low energies, the theoretical estimates are calculated in the chiral unitary model [9], which includes meson-exchange contributions and $s$ channel $N^{*}(1710)$ formation. They roughly agree with the data. For estimating the theoretical cross section at high energies, the matrix element of Eq. (2) should be calculated. However, it is not easy at this stage because there are a significant number of processes like Fig. 2 should be calculated, and the distribution amplitudes $\phi_{\pi, p, K^{0}, \Lambda(1405)}$ are not known. For a rough estimate, we use the experimental data of Fig. 6 and the counting rule. The obtained cross sections are shown in Fig. 7. If the $\Lambda$ (1405) is a five-quark state, the total number of constituents is $n=2+3+2+5=12$. The counting rule indicates the scaling $s^{10} d \sigma / d t=$ constant, and the cross section is shown by the solid curve. The dashed curve indicates the cross section if $\Lambda(1405)$ is a three-quark baryon. There is a clear difference between the curves, so that the internal structure of $\Lambda(1405)$ could be determined by observing the scaling behavior of the exclusive cross section.

We discussed only the $\Lambda$ (1405) production; however, the idea could be applied for other exotic hadron candidates. They could be experimentally studied at various experimental facilities including J-PARC, LEP, JLab, and CERN-COMPASS.

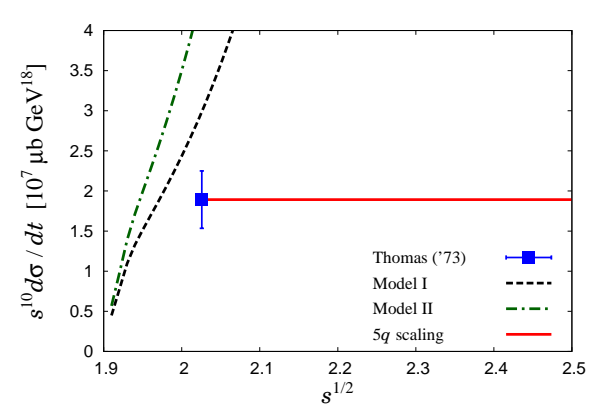

Fig. 6. Cross section of $\pi^{-}+p \rightarrow K^{0}+\Lambda(1405)[3]$.

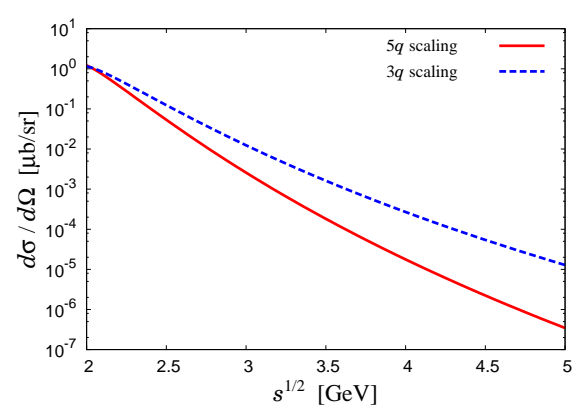

Fig. 7. Scaling of $\pi^{-}+p \rightarrow K^{0}+\Lambda$ (1405) cross section at high energies [3].

\section{Acknowledgement}

This work was partially supported by a Grant-in-Aid for Scientific Research on Priority Areas "Elucidation of New Hadrons with a Variety of Flavors (E01: 21105006)" from the ministry of Education, Culture, Sports, Science and Technology of Japan.

\section{References}

[1] R. H. Dalitz and S. F. Tuan, Ann. Phys. 10, 307 (1960); R. L. Jaffe, Phys. Rept. 409, 1 (2005).

[2] H. Kawamura and S. Kumano, KEK-TH-1589.

[3] H. Kawamura, S. Kumano, and T. Sekihara, Phys. Rev. D 88, 034010 (2013).

[4] G. P. Lepage and S. J. Brodsky, Phys. Lett. B 87, 359 (1979); Phys. Rev. D 22, 2157 (1980); A. V. Efremov and A.V. Radyushkin, Phys. Lett. B 94, 245 (1980).

[5] V. A. Matveev, R. M. Muradian, and A. N. Tavkhelidze, Lett. Nuovo Cim. 7, 719 (1973); S. J. Brodsky and G. R. Farrar, Phys. Rev. Lett. 31, 1153 (1973); Phys. Rev. D 11, 1309 (1975).

[6] C. White et al., Phys. Rev. D 49, 58 (1994); L. Y. Zhu et al., Phys. Rev. C 71, 044603 (2005).

[7] D. Rönchen et al., Eur. Phys. J. A 49, 44 (2013).

[8] D. W. Thomas, A. Engler, H. E. Fisk, and R. W. Kraemer, Nucl. Phys. B 56, 15 (1973).

[9] T. Hyodo et al., Phys. Rev. C 68, 065203 (2003). 